Gdańsk 2019, Nr. 41

https://doi.org/10.26881/sgg.2019.41.18

\author{
Marta Turska
}

Universität Gdańsk

https://orcid.org/0000-0002-3168-2716

\title{
Danziger Weinpreislisten. Zum Text im urbanen Raum
}

Der vorliegende Beitrag wird den im Zeitraum 1913-1938 in Danzig herausgegebenen Weinpreislisten gewidmet, die in der Bibliothek der Polnischen Akademie der Wissenschaften in Gdańsk aufbewahrt werden. Die an die Kunden verschickten Preislisten haben die Form mehrseitiger, teilweise reich verzierter gehefteter Broschüren mit kartoniertem Umschlag und sind als Texte mit dominierender informativer Funktion aufzufassen. Den Ausgangspunkt für die Analyse der Preislisten stellt der Katalog der Textualitätsmerkmale von DE BEAUgrande / Dressler (1981) dar. Das besondere Augenmerk gilt dem räumlichen und zeitlichen Kontext der Entstehung der Preislisten, der Einblick in die Danziger Wirklichkeit der jeweiligen Epoche gewährt und vermittelt, welche urbanen und kulturellen Elemente im kollektiven Gedächtnis der Einwohner der Stadt und in ihrer gemeinsamen Identitätsbildung eine Rolle spielten.

Schlüsselwörter: Textualitätskriterien, Weinpreislisten, Danzig, kollektives Gedächtnis, Erinnerungsräume

Danzig price lists of wine. On a text in the city space. - The topic of discussion of this paper are German price lists of wines and other alcoholic drinks of the years 1931-1938 from the collection of Gdańsk Library of the Polish Academy of Sciences. Wine price lists sent to clients were usually richly decorated brochures consisting of a few or over a dozen of pages and texts of clearly informative function. The initial point for the discussion are criteria of textuality according to DE BEAUGRANDE / DRESSLER (1981). The time-space context of the origin of the price lists is worth a particular attention, since it throws light on the epoch and its reality. It enables one to discover on what urban and cultural elements the collective memory and the collective identity of the inhabitants focused.

Keywords: criteria of textuality, Danzig, wine price lists, collective memory, spaces of memory

Gegenstand der vorliegenden Überlegungen sind die im Zeitraum 1913-1938 in Danzig herausgegebenen Weinpreislisten. Sie sind kein Danziger Spezifikum, denn solche Texte waren recht verbreitet und wurden überall im deutschsprachigen Raum von Weinhandlungen und Kolonialwarengeschäften an ihre Kunden versandt. Zwar wird der Name Danzig eher mit Spirituosen, wie Machandel (Wacholderschnaps) und Goldwasser (Gewürzlikör) sowie dem Bierbrauen in Verbindung gebracht, nichtsdestotrotz waren in der Zwischenkriegszeit auch treue Weinabnehmer in der Stadt vorhanden, was eben der Gegenstand der Untersuchung bezeugt.

Die Preislisten werden im Folgenden zuerst aus der Perspektive der Textlinguistik beleuchtet. In Anlehnung an den Katalog der Textualitätseigenschaften von DE BEAUgRande / 
Dressler (1981) wird versucht, die Situationszusammenhänge, in denen die Preislisten entstanden sind, zu schildern. Texte sagen etwas - sei es explizit oder implizit - über ihre Emittenten und Rezipienten aus und geben Einblick in die räumliche und zeitliche Dimension ihrer Entstehung.

Diese historische, im Hinblick auf ihren ,flüchtigen 'Charakter zweifelsohne zu den Ephemera gehörende Textsorte verfolgte ganz pragmatische Ziele. Jetzt aber, im zeitlichen Abstand von fast hundert Jahren, liefern uns die Preislisten interessante Informationen über das Danzig der 1920er und 1930er Jahre. Texte sind Medien kulturellen Gedächtnisses, Erinnerungsträger und Zeitzeugnisse, in denen sich der kulturelle Kontext ganzer Gesellschaften widerspiegelt. Sie ,konservieren' nicht nur Bilder der Vergangenheit, sondern geben auch Einblick in die Wirklichkeit der jeweiligen Epoche und werden dadurch zu einer Quelle des Wissens über das Selbstverständnis der Stadtgesellschaft, worauf im Weiteren eingegangen werden soll.

\section{Danziger Weinpreislisten aus dem Zeitraum 1913-1938: Darstellung des Korpus ${ }^{1}$}

In den Sammlungen der Danziger Bibliothek der Polnischen Akademie der Wissenschaften sind 17 Preislisten erhalten geblieben, die vorwiegend aus den 1920er und 1930er Jahren stammen. ${ }^{2}$ Eine Preisliste ist auf 1913/14 datiert, eine weitere enthält keine Hinweise auf das Herkunftsjahr. Zehn Preislisten (für die Jahre 1913/14, 1925, 1926, das Frühjahr 1927, den Herbst 1927, 1928, 1930, 1931/32, 1931/32, 1937/38) wurden von einer konkreten Firma, nämlich der Weingrosshandlung ${ }^{3}$ Carl Keller, Danzig, herausgegeben. Außerdem kommen je zwei Preislisten weiterer drei Firmen (der Weingrosshandlung. Spirituosen-Import R. Denzer - wahrscheinlich aus den Jahren 1926 und 1930, ${ }^{4}$ der Weingroßhandlung Daniel Feyerabendt - 1927 und 1933, der Weingrosshandlung Josef Fuchs - 1928 und 1929) vor. Darüber hinaus ist eine Preisliste der Firma Weingroßhandlung J.H.L. Brandt (ohne Jahresangabe) erhalten geblieben.

Die Preislisten haben die Form mehrseitiger, teilweise reich verzierter (worauf noch eingegangen wird) gehefteter Broschüren mit kartoniertem Umschlag. Es handelt sich hierbei um Gebrauchstexte, wobei ihre Grundfunktion eindeutig in der Information besteht: Sie sollen den Rezipienten über die Preise der erhältlichen Spirituosen und Neuigkeiten

\footnotetext{
1 Mein großer Dank gebührt der Mitarbeiterin der Bibliothek, Frau Aneta Kwiatkowska, die mein Interesse an den Preislisten geweckt und mir diese freundlicherweise zugänglich gemacht hat.

2 Außer den hier beschriebenen und analysierten Preislisten sind einige Auszüge aus den Preislisten erhalten geblieben, meistens in Form von zusammengefalteten ein- bis vierseitigen Flugblättern, die im Folgenden nicht berücksichtigt werden.

3 Die Namen werden in Originalschreibung angeführt.

4 Beide Preislisten beinhalten keine direkten Jahresangaben; die Jahresdaten können nur anhand des angegebenen Gründungsjahres der Firma und der Nummer der Preisliste (Nr. 73 und Nr. 76) sowie weiterer indirekter Indizien (wie die Aufschrift „75 Jahre Denzer Weine“ und „Goldene Medaille Grosse Ausstellung für Kochkunst, Hotel- und Gastwirtsgewerbe, Danzig 1926“) ermittelt werden.
} 
im Angebot der Weinhandlungen informieren. Den größten Teil der angebotenen Waren stellen Weine dar, die nach Herkunftsland bzw. -region ${ }^{5}$ gruppiert werden. Die stichwortartige Aufzählung der Weine unter Preis- und Mengenangabe begleiten Informationen über die Aufbewahrung, Behandlung und Serviertemperatur der Weine sowie den Ort der Flaschenabfüllung. In einigen Preislisten finden wir auch suggestiv formulierte Empfehlungen und Äußerungen zu den Eigenschaften und dem Geschmack der Weine (z.B. lebhaft; mild; elegant; blumig; zart; körperreich; grosser, edelsüßer Festwein; wuchtig, mit feiner Rasse und Süsse usw.). Darüber hinaus sind Verkaufs- und Zahlungsbedingungen sowie Informationen über Steuern und Skonto-Gewährung inbegriffen. Die Preislisten wurden, zumindest was die Stammkunden betrifft, per Post verschickt.

Die Sammlung ist mit 17 Exemplaren nicht besonders groß, aber nichtsdestotrotz als Quelle des Wissens über die soziale Wirklichkeit der Zwischenkriegsjahre sehr interessant. Da die Preislisten im vorliegenden Beitrag als Texte und Textsorte betrachtet werden, gilt es zuerst zu erklären, was unter einem Text zu verstehen ist.

\section{Weinpreislisten aus textlinguistischem Blickwinkel}

Wir haben es täglich mit den unterschiedlichsten Texten zu tun, und man versteht intuitiv sehr wohl, was unter dem Begriff, Text' zu verstehen ist. Erst die wissenschaftliche Beschäftigung mit Texten bringt die Schwierigkeit mit sich, den Untersuchungsgegenstand möglichst genau zu definieren und von ähnlichen, benachbarten Phänomenen abzugrenzen. Nach Duden-Universalwörterbuch (2003) ist Text eine „[schriftlich fixierte] im Wortlaut festgelegte, inhaltlich zusammenhängende Folge von Aussagen”, was wohl dem intuitiven Verständnis der Sprachbenutzer entspricht. Fest steht, dass Texte sprachliche Gebilde und „Form-Inhalt-Kopplungen“ sind (SChWARZ-Friesel / CONSTEN 2014: 10), wobei es von vielen Faktoren abhängt, ob sie auch als solche wahrgenommen werden. Es gibt Texte, die sofort als typische Repräsentanten der Kategorie Text erkannt werden, wohingegen andere weniger repräsentativ zu sein scheinen. ${ }^{6}$ In der engen Textauffassung werden Texte als „begrenzte Folge[n] von sprachlichen Zeichen mit charakteristischen Struktureigenschaften" (ebd. 16) und (meist) markierten Textbegrenzungssignalen definiert, als zusammenhängende, komplexe, d.h. die Satzebene überschreitende und schriftlich fixierte Einheiten, die der Übermittlung von zumindest zum Teil sprachlich kodierten Informationen dienen (vgl. ebd.).

Damit ist kurz umrissen, was unter Texten verstanden werden kann und womit sich die Textlinguistik u.a. beschäftigt. Auf die Textauffassung im weiteren Sinne, die die funktionale Perspektive berücksichtigt und jede, einen kommunikativen Zweck erfüllende Äußerung als Text deklariert, sowie auf die Diskussion um das Medium eines Textes (schriftlich

5 Das reiche Angebot umfasst u.a. Mosel-, Saar-, Ruwer-, Rhein- und andere deutsche Weine, elsässische Weine, Bordeaux-, Burgunder- und andere französische Weine, Tokajer und andere ungarische Weine, Champagner und andere Schaumweine, österreichische, spanische, portugiesische, griechische und italienische Weine, außerdem noch englische Biere, Cognac, Rum, Arrak, Whisky und verschiedene Liköre.

6 Zum Prototypenkonzept SANDig (2000), vgl. auch AdAmziK (2016: 40-42). 
vs. mündlich) ${ }^{7}$ wird hier nicht eingegangen, da dies für die in Druckform vorliegenden Preislisten, die ja den Gegenstand der folgenden Überlegungen bilden, irrelevant ist.

Um die in der Danziger Bibliothek der Polnischen Akademie der Wissenschaften aufbewahrten Weinpreislisten näher zu beleuchten, wird jedoch auf den Katalog der Textualitätsmerkmale von DE BEAUGRANDE / DRESSLER (1981) zurückgegriffen.

Nach AdAmziK (2016: 98) ist „die Suche nach einer bündigen Textdefinition inzwischen weitgehend ersetzt [...] durch die Arbeit mit einem Katalog von relevanten Texteigenschaften im Sinne einer explikativen Definition." Bestimmte Merkmale der Texte sollen demnach als Beschreibungsdimensionen begriffen werden (vgl. ebd.: 98). Die von DE BEAUGRANDE / Dressler (1981) vorgeschlagene Liste der Textualitätsmerkmale stellt immer noch, so ADAMZIK (2016: 98), einen besonders häufig gewählten Bezugspunkt dar. Nach DE BEAUGRANDE / Dressler (1981:3) ist Text „eine kommunikative Okkurrenz [...], die sieben Kriterien der Textualität erfüllt". Diesen strengen Kriterien würden viele sprachliche Äußerungen nicht entsprechen, die ihrer Kommunikativität wegen trotzdem als Texte fungieren (selbst in der Definition scheint die Kommunikativität "einer kommunikativen Okkurrenz" doch ein konstitutives Merkmal der Texthaftigkeit zu sein, vgl. AdAMzIK 2015: 161), deswegen werden die von DE BEAUGRANDE / DRESSLER vorgeschlagenen Textualitätsmerkmale in der späteren Literatur nicht mehr als obligatorische Kriterien der Textkonstitution, sondern als mehr oder weniger ausgeprägt vorliegende Eigenschaften (vgl. ADAmZıK 2016: 100) verstanden, die nur die Komplexität des Beschreibungsgegenstandes - des Textes - verdeutlichen und eine Art Checkliste für die Analyse darstellen (vgl. AdamziK 2015: 162). Diese Kriterien eignen sich sehr gut für die Beschreibung des Textes und eröffnen eine breitere Perspektive auf die Relationen zwischen dem Text und seinem außersprachlichen Kontext. Eine auf verschiedenen Beschreibungsebenen durchgeführte Analyse kann aufschlussreiche Befunde bezüglich des kulturhistorischen und sozialen Hintergrundes der Texte bringen.

Die Liste der Textualitätskriterien von DE BEAUGRANDE / Dressler (1981) eröffnen die textzentrierten Kriterien der Kohäsion, d.h. der grammatisch-lexikalischen Verknüpfungen an der Oberflächenstruktur des Textes, und der Kohärenz, d.h. seines inhaltlichen Zusammenhangs. Die Preislisten setzen sich aus unterschiedlichen Teiltexten zusammen, in denen sich die Kohäsions- und Kohärenzmittel unterschiedlich verteilen. Der Hauptteil der Preislistebroschüren, der meist aus einer Auflistung der Weine und anderer Spirituosen in einer Spalte und der Angabe der Preise in einer oder zwei weiteren (je nach Menge) besteht, kann wohl als ein nicht-kontinuierlicher Text (vgl. ADAmziK 2008: 363) betrachtet werden, in dem kaum Kohäsions- und Kohärenzsignale zu verzeichnen sind, anders als in den diesem Hauptteil angeschlossenen Verkaufsbedingungen, Zablungsbedingungen und Steuern, oder auch im in einigen Fällen vorangestellten Textteil Zur gefl. [gefälligen] Beachtung - in dem die Aufbewahrung, die Weinbehandlung und Serviertemperaturen der Weine, Biere und anderer Alkoholika besprochen werden, oder in den an den Kunden gerichteten persuasiven Ansprachen. ${ }^{8}$ An dieser Stelle sei nur festgestellt, dass die Preislisten kohärente und kohäsive

Dazu vgl. AdAmziK (2016: 70-76).

8 Wie z.B.: „Beifolgend erlaube ich mir, Ihnen meine neuste Preisliste mit der Bitte zu überreichen, bei eintretendem Bedarf sich meiner Firma freundlichst erinnern zu wollen. Hochachtungsvoll - J.H.L. Brandt“, 
Texte sind, das Augenmerk dieser Überlegungen gilt jedoch vor allem den verwenderzentrierten Kriterien.

Weitere Kriterien aus dem Katalog von De Beaugrande / Dressler wenden den Blick vom Text als Produkt ab und fokussieren auf die Textbenutzer und die räumlich-zeitliche Einbettung des Textes. Die Intentionalität als Lesbarkeit der Absicht des Senders, die er durch den Text zu vermitteln sucht, ist in diesem Fall eindeutig: Es geht um eine Werbemaßnahme, die Bekanntmachung der Kunden mit den angebotenen Produkten und Verkaufsförderung. Die Akzeptabilität, ein rezipientenzentriertes Merkmal, besagt, dass der Text vom Rezipienten mit einer bestimmten Erwartungshaltung gelesen wird; der Text muss dem Sprachgefühl und den Erwartungen der Empfänger (im Fall einer Preisliste solchen wie Überschaubarkeit, Vollständigkeit, aber auch Höflichkeit und Eleganz der Formulierung usw.) entgegenkommen. Die beiden Akteure - der Emittent und der Rezipient - werden noch im Weiteren in Verbindung mit der Situationalität zur Sprache gebracht. Mit Infor mativität ist das Informationspotential des Textes gemeint, d.h. das Verhältnis zwischen bekannten und unbekannten Informationen, das stark vom Kenntnisstand des Rezipienten abhängt und in Hinsicht auf historische Preislisten schwer zu deuten ist. Auf jeden Fall kann wohl behauptet werden, dass die Informationsvermittlung die wichtigste Aufgabe der Preislisten war.

Aus einer historischen Perspektive scheinen die Merkmale der Situationalität (Raum-Zeit-Konstellation) und Intertextualität (Bezug des Textes auf andere Texte) jedoch am aufschlussreichsten zu sein.

Die Situationalität ist die Korrelation mit natürlichen Umständen und der außersprachlichen Situation, in denen der Text funktioniert, wobei zwischen der Textproduktionssituation und der Textrezeptionssituation zu unterscheiden ist, weil Produktion und Rezeption meistens zeitlich und räumlich versetzt sind. Den Kern dieses Kriteriums bilden die im Weiteren noch aufgegriffenen Dimensionen der Zeit und des Raums. In diesem Rahmen sind aber auch die für die Interaktionsakteure, also für den Produzenten (in Verbindung mit Intentionalität) und den Rezipienten (in Verbindung mit Akzeptabilität) charakteristischen Merkmale und die sie beeinflussenden Umstände zu betrachten.

Die Analyse der Preislisten sagt uns nicht viel über die Interaktanten. Die Texte beinhalten keine direkten Informationen über Textempfänger, aber es ergibt sich aus ihrem Inhalt, dass die Preislisten sowohl an neue als auch an bereits bekannte Kunden gerichtet waren. Es handelt sich dabei um auf dem Gebiet der Freien Stadt Danzig wohnhafte Kunden, aber auch solche, die außerhalb wohnen oder verbleiben (darüber informieren speziell an sie gerichtete Verkaufsbedingungen und die Auskunft über im Falle der Spirituosenausfuhr zu zahlende Steuern). Die Preislisten wurden auf Deutsch verfasst, waren also in erster Linie für deutschsprachige Kunden oder zumindest für über Deutschkenntnisse verfügende Personen konzipiert. Der

oder aber: „Die freundliche Aufnahme, die meine vorjährige Herbstpreisliste allseitig gefunden hat, veranlaßt mich, schon jetzt einen Auszug aus meiner diesjährigen Preisliste herauszugeben, der unter Berücksichtigung meiner neuen Einkäufe eine sorgfältige Auswahl besonders empfehlenswerter, auserlesener Weine und Spirituosen enthält. Es würde mich freuen, wenn Sie bei Ergänzung Ihres Bedarfs meinem heutigen Angebot Ihre geschätzte Aufmerksamkeit schenken würden. Meine Hauptpreisliste, ein ausführliches Verzeichnis meiner reichen Bestände enthaltend, steht Ihnen auf Wunsch jederzeit zur Verfügung. Hochachtungsvoll - Carl Keller. Preisliste PR 57. Danzig, im April 1927. 

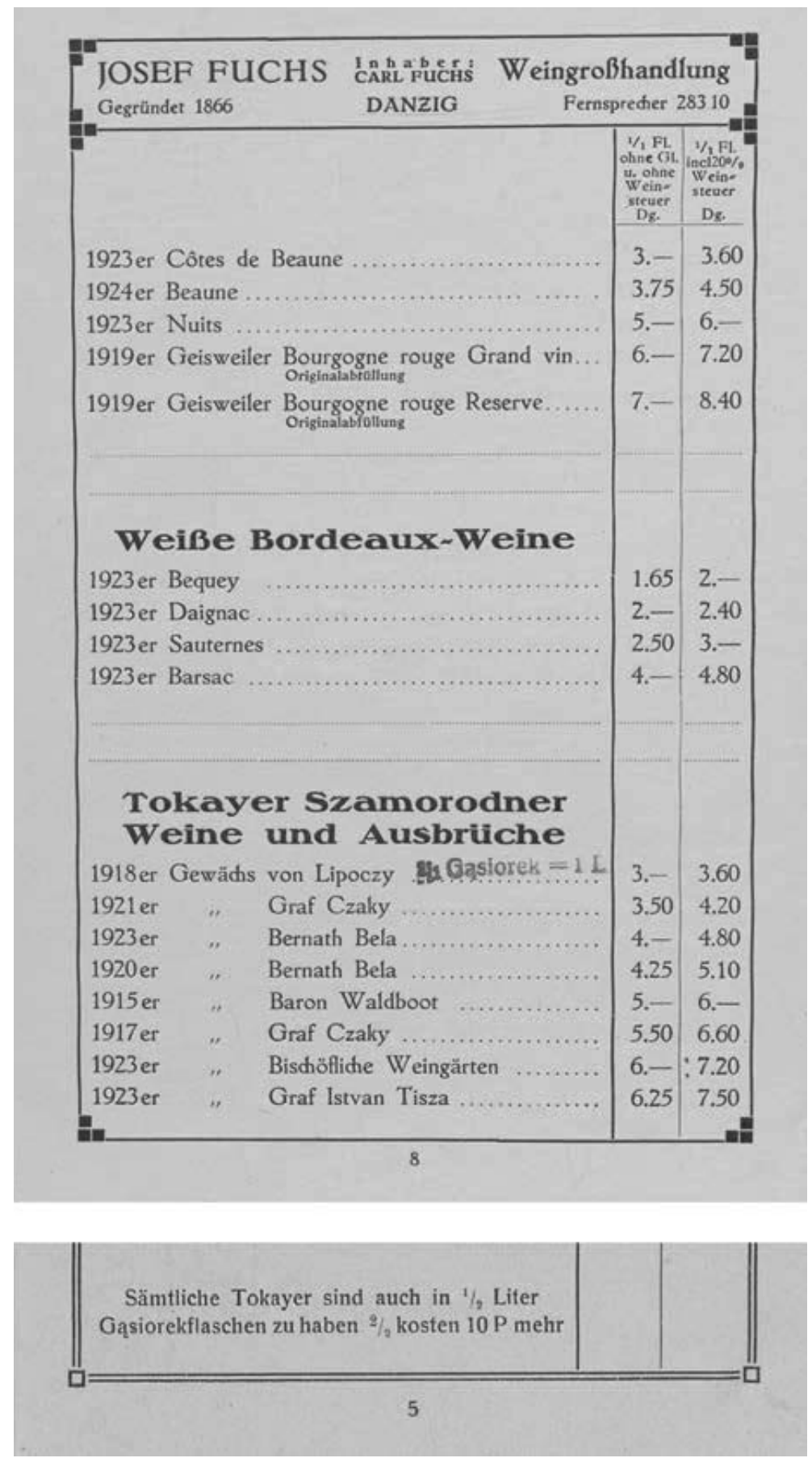

Abb. 1: Gąsiorekflaschen: Preislisten: Wein-Großhandlung Karl Josef Fuchs, 1928, S. 8 (Sign. Od $114814^{0} / 14$ ) und 1929, Fragment der S. 5 (Sign. Od $\left.114824^{0} / 7\right)$. 
Versuch, nur anhand der Preislisten generelle Schlussfolgerungen über die Sprachverhältnisse in der Freien Stadt Danzig zu ziehen, wäre natürlich etwas gewagt, sie bezeugen aber doch, dass die vorwiegend deutschsprachige Stadtgemeinschaft im Alltag durchaus auch mit polnischsprachigen Personen zu tun hatte. In zwei Preislisten ist nämlich die Bezeichnung „Gąsiorekflaschen“ (Abb.1) zu finden (als Angabe des Fassungsvermögens der Flaschen, aus dem Polnischen gasiorek, Glasballon'), was heißt, dass diese zumindest in bestimmten Kontexten geläufig war und es im alltäglichen Kontext zu deutsch-polnischen Sprachkontakten kam.

Von den Preislisten ausgehend (also sie als einzige Wissensquelle betrachtend), erfährt man ebenfalls nicht viel über die Textproduzenten. Es konnten die Geschäftsbetreiber selbst sein, deren Namen, Adressen, Telefonnummern angegeben werden, aber auch ihre Angestellten oder sonstige Beauftragte. Eine Preisliste wurde mit dem Logo der jeweiligen Werbeagentur versehen, möglicherweise wurden hier aber auch andere Texte in Auftrag gegeben. Auf den Umschlägen finden wir auch die Namen und Signets der Drucker. Die Kontaktangaben bringen uns die Danziger Gesellschaft dieser Zeit näher, erlauben uns die Texte im städtischen Raum zu platzieren und die Stadttopographie zu rekonstruieren.

Den Kern des Situationalitätsmerkmals bildet die Raum-Zeit-Konstellation. In historischer Perspektive werden die Preislisten zu Texten, in denen sich die Zeit und vor allem der Ort ihrer Entstehung - die Stadt Danzig - widerspiegeln. Das Kriterium der Intertextualität bedeutet in seiner weiten Auffassung, dass der konkrete Text Vertreter einer bestimmten Textsorte ist und auf andere Texte dieser Sorte Bezug nimmt. Was jedoch im Falle von Preislisten vielleicht überraschen kann, sind intertextuelle Bezüge in der engen Auffassung, also Bezüge auf literarische Texte. Beide Kriterien werfen Licht auf den kulturhistorischen Hintergrund der Texte und eröffnen neue Interpretationsmöglichkeiten in Bezug auf die Stadt und die städtische Gemeinschaft.

\section{Die Stadt als Kommunikationsgemeinschaft}

Der urbane Raum stellt eine Ganzheit dar, die Menschen und Orte ausmachen. Nach Wallis (1990: 45) ist die Stadt ein System, das sich auch aus zwei Subsystemen - einem urbanistischen und einem gesellschaftlichen - zusammensetzt, welche nach dem Prinzip der Rückkoppelung zusammenwirken, dennoch aber autonom bleiben. ${ }^{9}$

Unter dem urbanistischen Subsystem werden das Gesamtbild der materiellen, vom Menschen geschaffenen Elemente der Stadt sowie jegliche natürliche Elemente verstanden, die ihre räumliche Struktur prägen. Das urbanistische Subsystem umfasst die räumliche Verteilung aller innerhalb einer Stadt zu erfüllenden Grundfunktionen, d.h. die Verteilung der Wohnorte, Arbeitsplätze, Bildungs- und Wissenschaftseinrichtungen, der Verwaltung, des Handels, der Dienstleistungen, der Erholung, des Sports, des gesellschaftlichen und künstlerischen Lebens, der Kulthandlungen. Jede Zone und jedes Stadtviertel hat ein eigenes architektonisches, urbanes und funktionales Bild, und alle sind in unterschiedlichem Maße attraktiv.

9 „Miasto jest systemem złożonym z dwóch organicznie powiązanych, współdziałających na zasadzie sprzężeń zwrotnych, lecz autonomicznych podsystemów - urbanistycznego i społecznego" (WALLIs 1990: 45). 
Das gesellschaftliche Subsystem umfasst die Gemeinschaft der Stadtbenutzer, d.h. vor allem - aber nicht nur - ihre Einwohner. Eine Metropole macht vor allem „der freiwillige Zustrom aus allen Himmelsrichtungen" aus (HOFFMANN-AXTHELM, zit. nach LiNDNER 2011: 386), weil sie den Zuwanderern als der Ort eines vergrößerten Möglichkeitshorizontes gilt (vgl. LINDNER 2011:386), wo neue Lebensstile und „Großstädter mit einer neuen mentalen Ausstattung, mit einem neuen psychischen Sensorium "(ebd. 387) entstehen. Die Stadtgemeinschaft bewirtschaftet das urbanistische Subsystem und schafft dadurch die funktionale Raumgemeinschaft oder den sozialen Stadtraum (vgl. Grotex 2018: 165, WALlis 1990: 45 und 101).

Beide Subsysteme werden durch eine Kette historischer Ereignisse geprägt, deren Mitwirkung immer durch ihre Vergangenheit bedingt ist (vgl. Wallis 1990: 45). Die räumliche Verteilung der materiellen Infrastruktur macht es der Stadtgemeinschaft möglich, diese unterschiedlich zu nutzen sowie diverse räumliche Verhaltensweisen auszuführen. Die jahrhundertelange Tradition mancher Verhaltensweisen und gewisse Gewohnheiten in diesem Bereich verleihen den unterschiedlichen Stadtelementen kulturellen Wert und historisch fundierte symbolische Bedeutung:

„Für die Bewohner bildet die Stadt einen Vorstellungsraum, der den physikalischen Raum insofern überlagert, als er der durch die begleitenden Bilder und Symbole hindurch erlebte und erfahrene Raum ist. Diese tatsächliche, weil gelebte Stadt bildet eine labyrinthische Wirklichkeit, eine totale soziale Erfahrung." (LINDNER 2011:392)

Der Stadtorganismus wird herausgebildet und kann dank dem kommunikativen Faktor reibungslos funktionieren. Durch Kommunikation, durch soziale und kulturelle Teilhabe, entsteht das Kollektive. Als Ergebnis der Kommunikationsprozesse konstituiert sich die Identiät einer Gemeinschaft, ihr Selbstbewusstsein in Bezug auf sich selbst, auf ihre Lebenssituation und soziale Zugehörigkeit. Nach Lindner (2011:392) ist das Gedächtnis der Stadt, „verstanden als die unbewusste Organisation aller vergangenen und gegenwärtigen sozialen Praktiken und der mit ihnen verknüpften geistigen oder symbolischen Bedeutungen“", das, was die Stadt davor bewahrt, ihre Identität zu verlieren. In Kommunikationsprozessen bildet sich im physischen Stadtraum „der gemeinsame Raum der Erlebnisse, und dadurch der gemeinsame Raum des Gedächtnisses und der Erinnerungen "10 (GROTEK 2018: 166, Übers.: M.T.) heraus. Im Raum der gemeinsamen Erfahrungen und Erlebnisse funktionieren gewisse topographische Orte, Ereignisse oder aber auch Gestalten und Persönlichkeiten, Texte und Handlungen als Erinnerungsorte im Sinne von Pierre Nora (1998). In der breiteren Auffassung von Jan und Aleida Assmann entstehen „durch jene partielle Ausleuchtung von Vergangenheit, wie sie ein Individuum oder eine Gruppe zur Konstruktion von Sinn, zur Fundierung ihrer Identität, zur Orientierung ihres Lebens, zur Motivierung ihres Handelns brauchen“ nicht nur Erinnerungsorte, sondern ganze Erinnerungsräume (Assmann 1999: 408; vgl. GROTEK 2018: 168). Um die Erinnerungsorte und -räume konzentriert sich das kollektive Gedächtnis verschiedener Gruppen und Generationen. ${ }^{11}$

10 „[...] wspólna przestrzeń przeżyć, a co za tym idzie, pamięci i wspomnień” (GROTEK 2018: 166).

11 Der Begriff des kollektiven Gedächtnisses wurde von dem französischen Sozialwissenschaftler Maurice HALBWACHS (1985) eingeführt. 


\section{Lokale Erinnerungsräume}

Nach HalBWACHS (1985: 142) ,gibt es kein kollektives Gedächtnis, das sich nicht innerhalb eines räumlichen Rahmens bewegt". In den Danziger Weinpreislisten finden wir bestimmte Elemente, die uns das Zusammenspiel des Räumlichen und der Urbanität einerseits und des Erinnerungsdiskurses andererseits näherbringen.

Die direkte verbale Aussage der Preislisten richtet sich auf den Vertrieb von Weinen und Spirituosen. Diese Aussage wird jedoch durch eine indirekte begleitet, die sich unter anderem in der Text- und Bildgestaltung äußert. Neben der informativen Grundfunktion hat der Text ebenfalls eine appellative Funktion, d.h. der Produzent versucht mit dessen Hilfe, den Rezipienten zu einer Handlung zu bewegen. Die graphische Gestalt des Textes soll seine direkte Aussage stärken, das Gefühl für Ästhetik des Rezipienten, dessen Sensibilität und Emotionalität ansprechen. Bedeutungsvoll ist die Wahl der graphischen Elemente, in der sich die kollektive Identität der Stadtgemeinschaft von Danzig niederschlägt.

Einige Preislisten wurden zu diesem Zweck mit ornamentalen Schmuckmotiven, andere wiederum mit graphischen Motiven versehen, die das Herkunftsland des betreffenden Weins oder zumindest die Gegend, aus der der jeweilige Wein stammte, darstellen. Diese sorgfältige, geschmackvolle Text- und Bildgestaltung soll positive Assoziationen wecken und die Aufmerksamkeit des Kunden gewinnen.

Ein besonderes Interesse gilt hier jedoch den Preislisten, deren bildliche Komponenten sich auf die Elemente der materiellen Stadtinfrastruktur beziehen, die eine besondere Bedeutung und einen symbolischen Wert für die Identität der kommunikativen Stadtgemeinschaft haben. Mehrere Preislisten sind mit dem Stadtwappen versehen, andere zeigen die wichtigsten Bauwerke Danzigs, um die sich das kollektive Gedächtnis der Stadtgemeinschaft konzentriert, die für die Konstruktion der urbanen Identität der Stadtbewohner, abgesehen von ihrer nationalen Zugehörigkeit, wichtig sind.

Interessant ist in diesem Zusammenhang die Preisliste von Daniel Feyerabendt (1927) (Abb. 2), die mehrere Danziger Sehenswürdigkeiten gleichzeitig zeigt: Zum einen sehen wir das Danziger Krantor, das größte im mittelalterlichen Europa, daneben das Haus der Naturforschenden Gesellschaft. In der unteren Reihe sieht man die Darstellungen des Rechtstädtischen Rathauses und die imposante Silhouette der Marienkirche. Jedes dieser Bauwerke allein kann als Wahrzeichen der Stadt betrachtet werden und deren Macht und Bedeutung bezeugen.

Eine weitere erhalten gebliebene Preisliste von Daniel Feyerabendt aus dem Jahr 1933 (Abb. 3) zeigt ein Fragment des heute nicht mehr existierenden (1946 laut Beschluss des Nationalen Stadtrates von Gdańsk beseitigten) Kriegerdenkmals am Holzmarkt (heute Targ Drzewny), das den in den der Reichsgründung vorangegangenen Kriegen (1864-1870) gefallenen Soldaten gewidmet war. Im Hintergrund sieht man den Sitz der Weingroßhandlung (die den Weinpreislisten zu entnehmende Adresse lautet: Am Holzmarkt 8).

Anhand der nur zwei erhalten gebliebenen Preislisten von Daniel Feyerabend darf man zwar keine allgemeingültigen Schlüsse ziehen, aber das Danzigtum scheint ein wichtiges Merkmal seiner Werbestrategie zu sein; zum einen werden durch das Gründungsdatum (und die Weingroßhandlung von Daniel Feyerabend ist laut Preisliste die älteste in Danzig, gegründet 1747) Ansässigkeit, Tradition und Dauer unterstrichen, zum anderen - die Bindung an die Stadt selbst. 


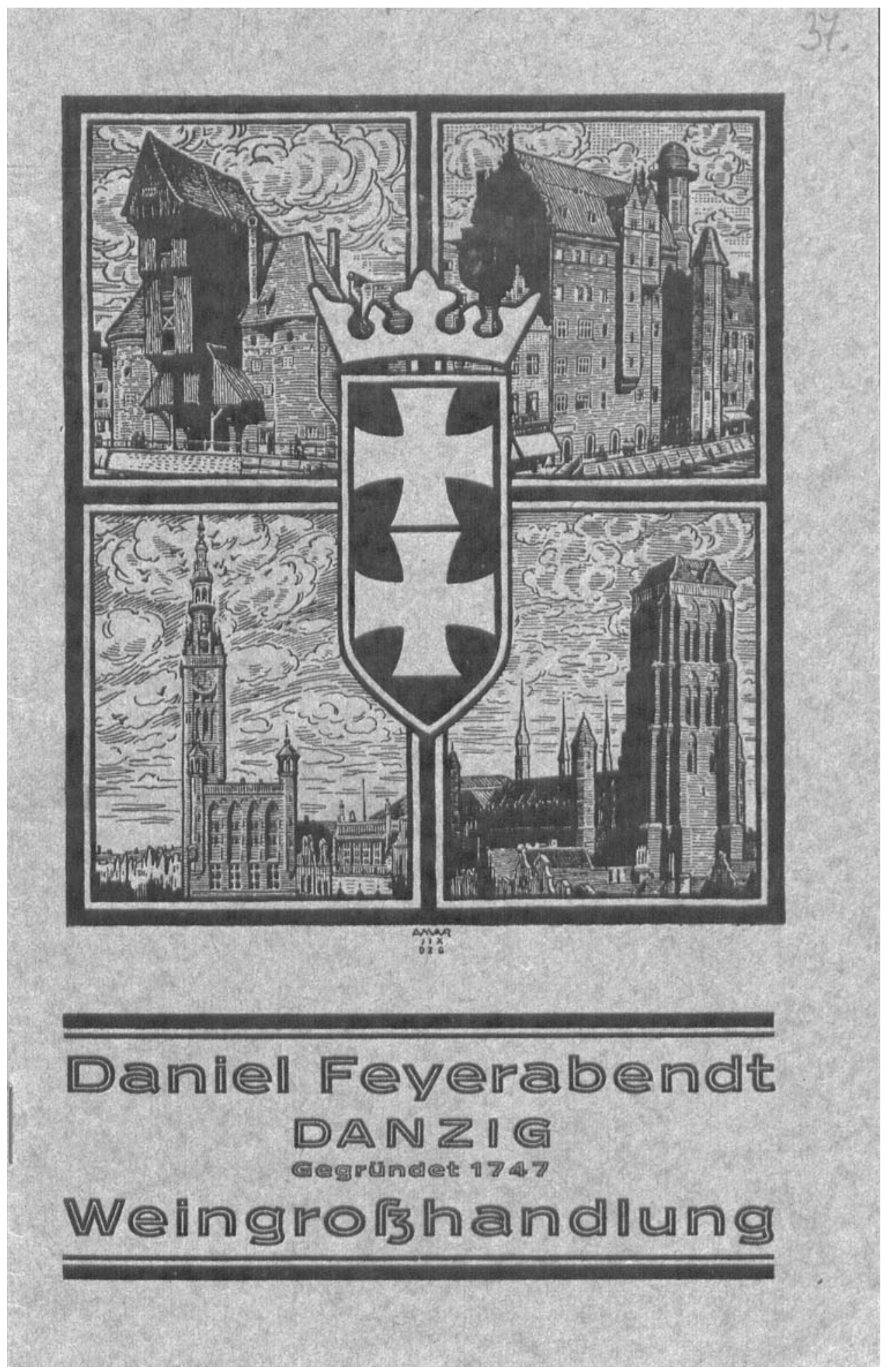

Abb. 2: Preisliste Weingroßhandlung Daniel Feyerabendt, 1927, Titelseite (Sign. Od 11480 4º/37) 


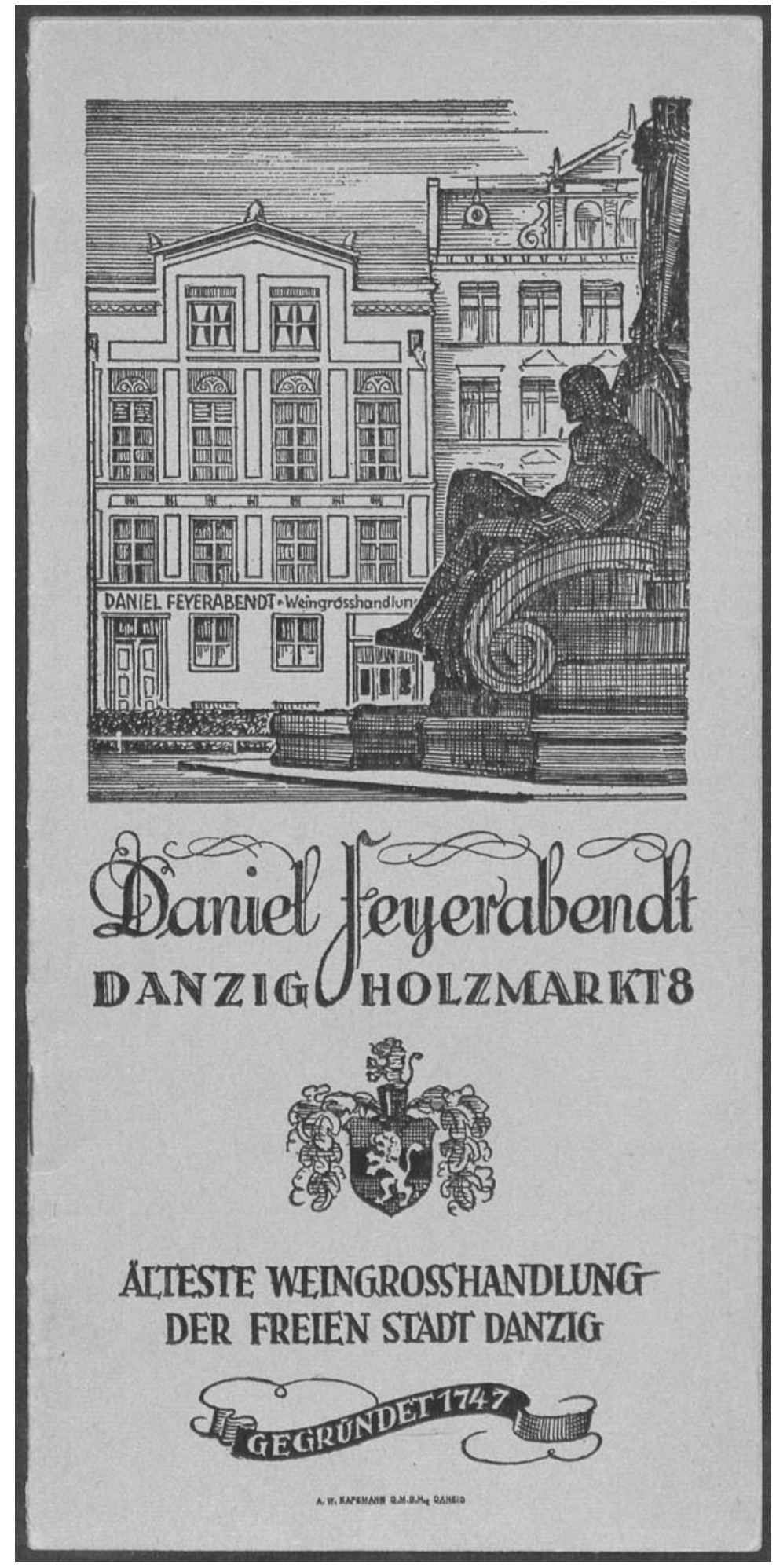

Abb. 3: Preisliste Weingroßhandlung Daniel Feyerabendt, 1933, Titelseite (Sign. Od $114804^{0} / 46$ ) 
Einen Blick auf die Marienkirche und die Frauengasse unter dem Frauentor, die zu den bekanntesten Danziger Aussichten gehören, finden wir in der Preisliste von J.H.L. Brandt (ohne Jahresangabe) (Abb. 4).

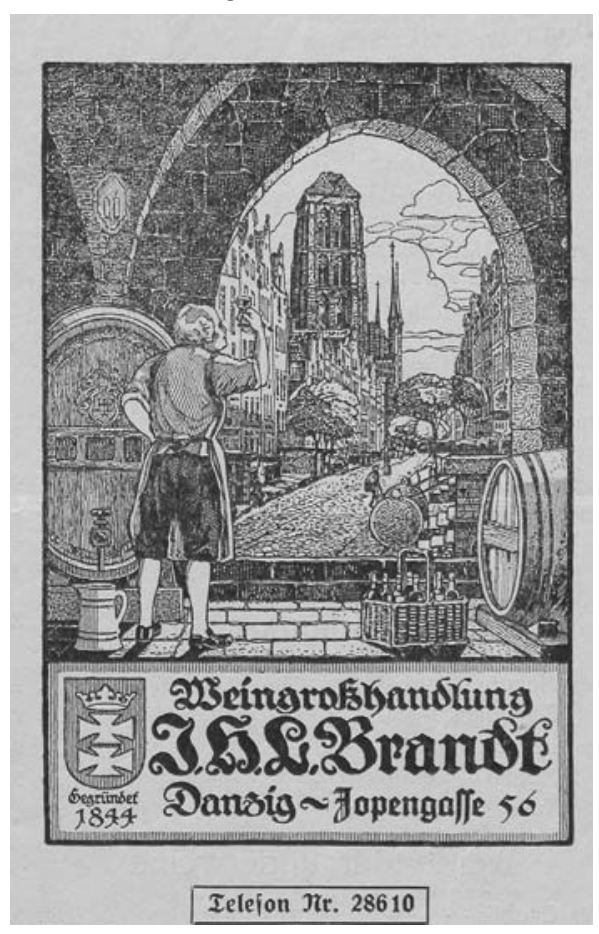

Abb. 4: Preisliste Weingroßhandlung J.H.L. Brandt (ohne Jahresangabe), Titelseite (Sign. Od $114824^{0} / 6$ )

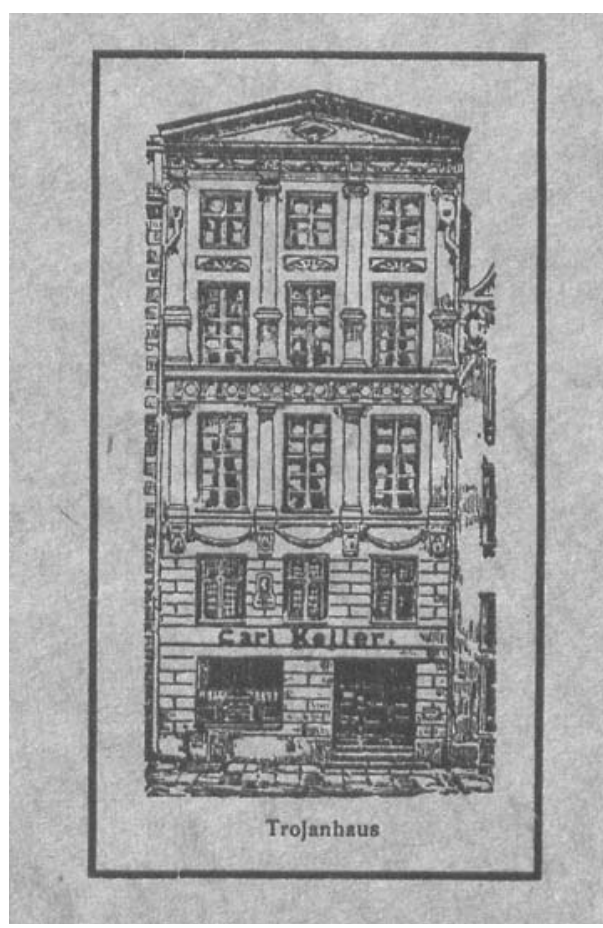

Abb. 5: Preisliste Weingrosshandlung Carl Keller, 1913/14, ein Fragment der Rückseite des Umschlags (Sign. Od $114824^{0} / 8$ )

Die Weingroßhandlung von Carl Keller befand sich unter der Anschrift: Hundegasse 101, in jenem Haus, in dem auch der deutsche Schriftsteller Johannes Trojan (1837-1915) geboren wurde. Seine Kariere wurde wohl in Danzig, seiner Heimatstadt, mit Interesse verfolgt. 1909 wurde eine der neuen Straßen der Stadt nach ihm benannt. ${ }^{12}$ Das sogenannte Trojanhaus wurde zum graphischen Leitmotiv seiner Werbemaßnahmen. Auf der ältesten erhalten gebliebenen Preisliste aus den Jahren 1913/14 sieht man das Trojanhaus auf der Rückseite des Umschlags (Abb. 5). In den 1920er Jahren rückt es nach vorne und wird immer geschmackvoller sowie ausgefallener gestaltet (Abb. 6).

In den Preislisten der Weingroßhandlung von Carl Keller finden wir auch weitere intertextuelle Bezüge auf deutsche und deutschsprachige Literatur: Es gibt Zitate aus dem Schaffen von Georg Engel (1866-1931) (Preisliste 30/31) und Ernst von Wolzogen (1855-1934) (Preisliste 31/32). Entweder war der Emittent der Preislisten ein Liebhaber der deutschen

12 Vgl. EK [Edmund Kotarski]: Trojan Johannes, in: Gedanopedia, https://www.gedanopedia.pl/? title=TROJAN_JOHANNES (25.11.2018). 
Literatur und ließ in den Werbemaßnahmen im Rahmen seiner Erwerbstätigkeit seinen persönlichen Interessen freien Lauf, oder aber er zielte auf die Unterstreichung der Bindung an die deutsche Literatur und Kultur sowie das Deutschtum im Allgemeinen ab. ${ }^{13}$ Diese letzte These würde vielleicht noch von einer anderen wichtigen Tatsache untermauert, nämlich der Verwendung der Frakturschrift in der Preisliste aus den Jahren 1937/38 (Abb. 7). Die bisherige Antiqua auf der Titelseite und in Aufschriften wurde durch die Fraktur ersetzt, was wohl auch als signum temporis gedeutet werden kann, das mit dem erneuten Aufleben der Fraktur, besonders als Aufzeichnungsschrift, die im Zeitraum 1933-1941 in den Dienst des Nationalsozialismus gestellt wurde, verbunden war. ${ }^{14}$ Die gebrochene Schrift galt als „arteneigene" deutsche Schrift (vgl. Stiebner / Leonhard 1977: 57), ${ }^{15}$ wahrhaftig deutsch und diente wahrschein-

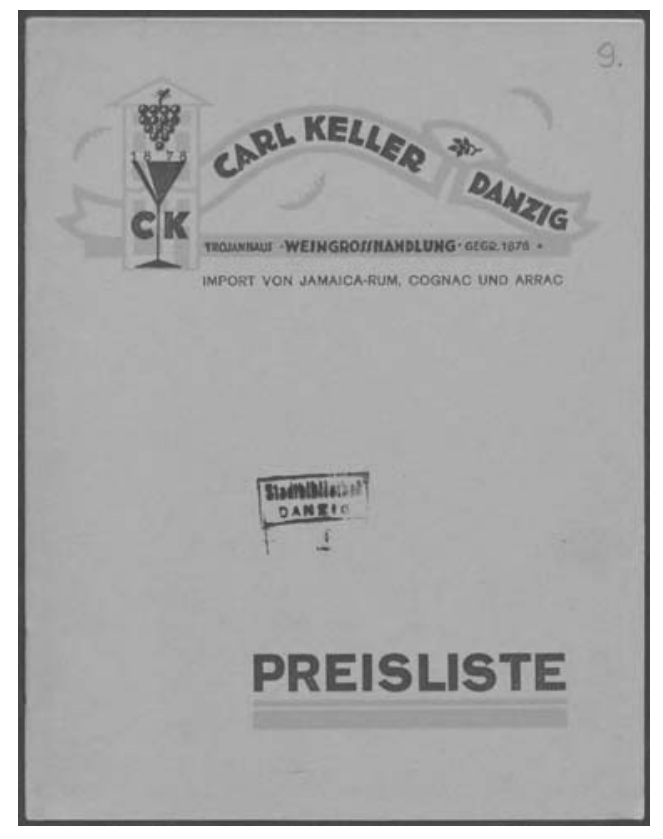

Abb. 6: Preisliste Weingrosshandlung Carl Keller, 1926, Vorderseite des Umschlags (Sign. Od $114804^{\circ} / 9$ ) lich, so wie auch die Zitate aus der deutschsprachigen Literatur, der Unterstreichung der Zugehörigkeit zum deutschen Sprach- und Kulturraum.

Anhand der Preislisten wird deutlich, wie die Integration von Schrift und Bild, von verbalen und visuellen Elementen das informative, ästhetische und persuasive Potenzial des Textes stärkt. Der Einsatz von visuellem Material und typographischen Mitteln lässt sich einfach begründen: Bilder „bewegen“, unabhängig von der Gattung und vom Medienträger (BREDEKAMP 2007, nach BIELAK 2012: 14). Visuelle Überlieferungen dienen als Residuum des Wissens über Kultur. Die Distanz zur Vergangenheit wird verkürzt (vgl. BIELAK 2012: 10), wenn man die visuellen Elemente in das Forschungsfeld der Textlinguistik einfügt. Noch einmal wird im Kontext des bildlichen Materials auf die Rolle des Emittenten verwiesen: Nach Jan Assmann ist das im visuellen Material fixierte Abbild der Wirklichkeit Gegenstand der Reflexion des Emittenten, der als teilnehmender Beobachter Fragmente der umgebenden Wirklichkeit auswählt, um sie zu fixieren, und so wird er zum bei deren Schaffung engagierten Zeugen. So nimmt er auch an der Speicherung des gemeinschaftlichen und individuellen Gedächtnisses teil (vgl. BIELAK 2012: 15).

13 Obwohl schon einige Jahre später, während des NS-Regimes, Georg Engel als Nicht-Arier eingestuft wurde und man seine Werke verbrannte.

14 Die gotischen und Frakturschriften wurden wenige Jahre später zu „Schwabacher Judenlettern“ deklariert und 1941 wurde ihre Anwendung untersagt (STIEBNER / LEONHARD 1977: 57).

15 Für die Literaturhinweise und freundliche Auskunft zur Geschichte der Typographie sei Herrn Dr. Adam Gorlikowski gedankt. 


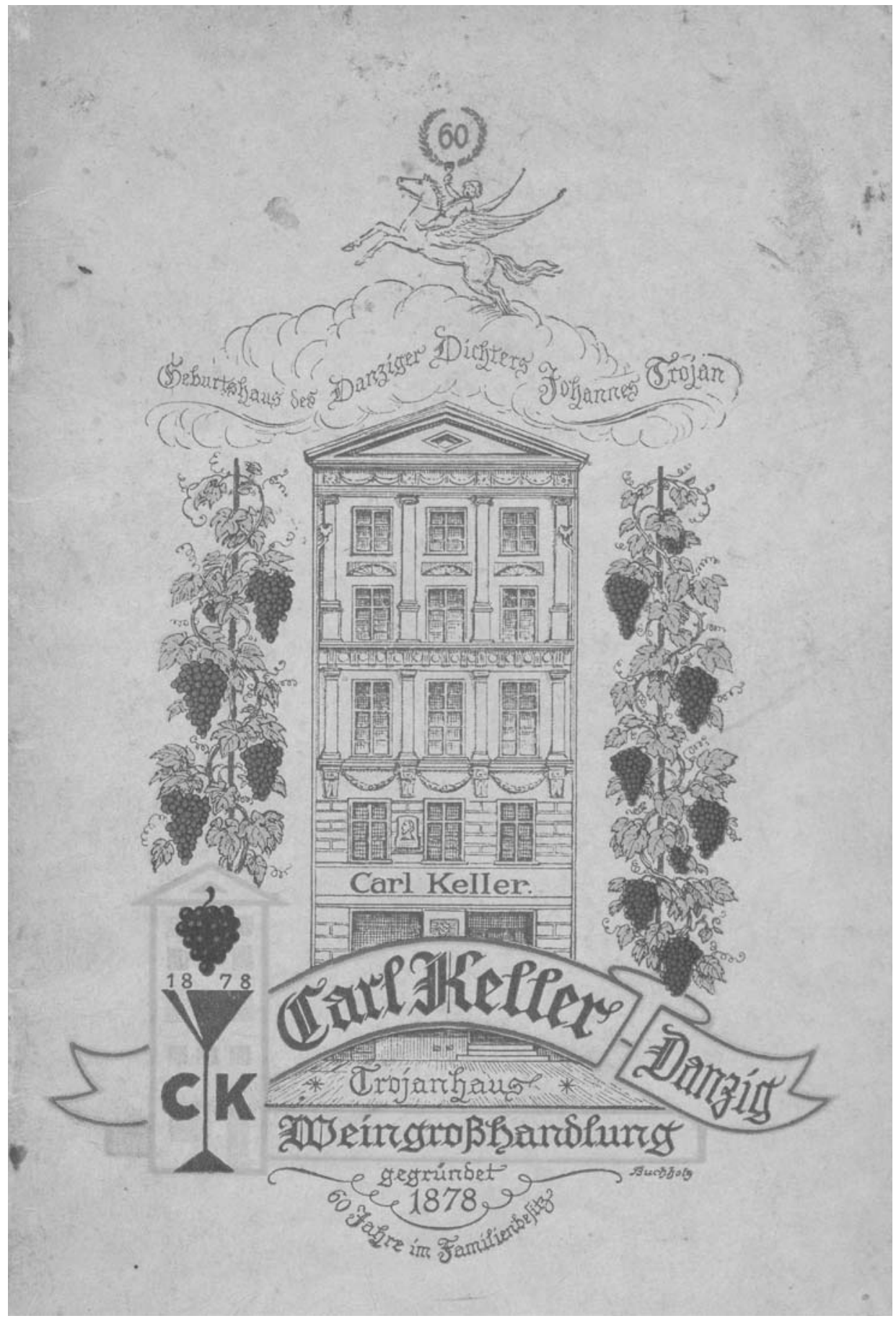

Abb. 7: Preisliste Weingrosshandlung Carl Keller, 1937/38, Vorderseite des Umschlags (Sign. C167c) 


\section{Fazit}

Die Stadt ist ein sozial konstruierter narrativer Raum, in dem unterschiedliche Texte entstehen. SZALEWSKA (2017: 265) definiert die Stadt als „einen wirklichen geographischen Raum, innerhalb dessen Textualität und Materialität, Bild und Wort, und letztendlich - Orte und Literaturen zusammenwirken" (Übers.: M.T.). ${ }^{16}$ Die in diesem Beitrag besprochenen, kurz vor dem Ersten Weltkrieg bis in die späten 1930er Jahre in Danzig entstandenen und heute in der Danziger Bibliothek der Polnischen Akademie der Wissenschaften aufbewahrten Weinpreislisten können als Texte angesehen werden, die in einem konkreten räumlichen Rahmen entstanden sind und diesen Rahmen widerspiegeln. Die verbalen und visuellen Informationen der Preisbroschüren ergänzen sich gegenseitig, die sich aus ihrer Integration ergebende Synergie fördert die Wirkung der Texte und verlockt zur Suche nach verborgenen Bedeutungen und äußeren Zusammenhängen.

Aus historischer Perspektive werden die Texte zu einer Quelle von Information über die Kommunikationsgemeinschaft, die sie betreffen. Sie vermitteln, welche konkreten Orte und Artefakte im Stadtraum im kollektiven Gedächtnis seiner Einwohner eine Rolle spielen, welche das Identitätsgefühl der Gemeinschaft ansprechen beziehungsweise stiften. Als Produkte menschlichen Geistes und soziokulturelle Phänomene sind die Texte in ihren jeweiligen außersprachlichen Kontext eingebettet, den die Stadt Danzig bildet, „,in Mythen schaffender Ort“, ein „Danzig, das es nicht mehr gibt“ (LoEw 2006, zit. nach BIELAK 2012: 15 in der Übers. von Józef Jarosz). Auch Ephemera, flüchtige, für einen Augenblick gedachte Texte häufen sich mit der Zeit an, bis sie „eine Textur, ein Gewebe bilden, in dem die Stadt im wahrsten Sinne des Wortes ,verstrickt" ist“" (LINDNER 2011: 396), auch sie können zur Erforschung von gesellschaftlichen Situationen und Verwandlungen des Gedächtnisses dienen.

\section{Quellen: Preislisten mit Signaturen}

Weingrosshandlung Carl Keller, Danzig, Engros-Flaschen-Preisliste Nr. 36, 1913/14 - Sign. Od 11482 4\%/8 Weingrosshandlung Carl Keller, Danzig, Preisliste PR 46, Herbst 1925 - Sign. Od 11480 4\% 12 Weingrosshandlung Carl Keller, Danzig, Preisliste PR 54, Herbst 1926 - Sign. Od $114804^{\circ} / 9$ Weingrosshandlung Carl Keller, Danzig, Preisliste PR 57, im April 1927 - Sign. Od 11480 4\% 10 Weingrosshandlung Carl Keller, Danzig, Preisliste PR 59, Herbst 1927 - Sign. Od 11480 4\% 11 Weingrosshandlung Carl Keller, Danzig, Preisliste PR 62, Herbst 1928 - Sign. Od 11481 4\% 16 Weingrosshandlung Carl Keller, Danzig, Preisliste PR 68, Frühjahr 1930 - Sign. Od 11483 4\%/3 Weingrosshandlung Carl Keller, Danzig, Preisliste D PR 73, 1930/31 - Sign. Od 11490 2\%/15 Weingrosshandlung Carl Keller, Danzig, Preisliste D PR 77, 1931/32 - Sign. Od 11483 4\%/4 Weingrosshandlung Carl Keller, Danzig, Preisliste D PR 106, 1937/38 - Sign. C 167 c Weingrosshandlung. Spirituosen-Import R. Denzer, Nr. 73 - Sign. Od 11480 4\%34 Weingrosshandlung. Spirituosen-Import R. Denzer Nr. 76 - Sign. Od 11483 4\% 1

16 „[Miasto rozumiemy jako] rzeczywistą przestrzeń geograficzną, w której obrębie dochodzi do współdziałania tekstualności i materialności, obrazu i słowa, wreszcie - miejsca i literatury" (SzALEwsKA 2017: 265). 
Weingroßhandlung Daniel Feyerabendt, 1927 - Sign. Od $114804^{\circ} / 37$

Weingroßhandlung Daniel Feyerabendt, 1933 - Sign. Od $114804^{\circ} / 46$

Weingrosshandlung Josef Fuchs, 1928 - Sign. Od $114814^{\circ} / 14$

Weingrosshandlung Josef Fuchs, 1929 - Sign. Od $114824^{\circ} / 7$

Weingroßhandlung J.H.L. Brandt - Sign. Od $114824^{\circ} / 6$

\section{Bibliographie}

Adamzik, Kirsten (2008): Der virtuelle Text oder: Die Rolle der Sprachgemeinschaft für die Herstellung von Textualität. In: Zeitschrift für germanistische Linguistik vol. 36.3, 355-380, https://www. unige.ch/lettres/alman/files/1115/1100/3727/adamzik-2008-zgl.pdf(14.11.2018).

Adamzik, Kirsten (2015): Das Wort im Text. In: Hass, Ulrike / Storjohann, Petra (Hg.): Handbuch Wort und Wortschatz. Berlin: de Gruyter, 152-174.

Adamziк, Kirsten (2016): Textlinguistik. Grundlagen, Kontroversen, Perspektiven. Berlin: de Gruyter.

Assmann, Aleida (1999): Erinnerungsräume. Formen und Wandlungen des kulturellen Gedächtnisses. München: Beck.

Assmann, Jan (2007): Das kulturelle Gedächtnis. Schrift, Erinnerung und politische Identität in frühen Hochkulturen. München: Beck.

BIELAK, Jacek (2012): Fotografia jako medium obrazowe i przedmiot badań/Fotografie als bildliches Medium und Forschungsgegenstand. In: Rudolf Th. Kubn (1842-1900) - fotografkrajobrazu, architektury i morza/ Rudolf Th. Kuhn (1842-1900) - Fotograf der Landschaft, Architektur und Ostsee. Übers. von Józef Jarosz. Gdańsk: Fundacja Karrenwall; Toruń: Wydawnictwo VIA, 41-44.

BREDEKAmp, Horst (2007): Bilder bewegen. Von der Kunstkammer zum Endspiel. Berlin: Wagenbach.

BRINKER, Klaus (2010): Linguistische Textanalyse. Eine Einführung in Grundbegriffe und Methoden. 7., durchges. Aufl. Berlin: Schmidt.

De Beaugrande, Robert-Alain / Dressler, Wolfgang Ulrich (1981): Einführung in die Textlinguistik. Tübingen: Niemeyer.

EK [Kotarski, Edmund]: Trojan Johannes. In: Gedanopedia, https://www.gedanopedia. $\mathrm{pl}$ /?title=TROJAN_JOHANNES (25.11.2018).

GroteK, Edyta (2015): Dziewiętnastowieczny Toruń wspólnotą języka / językową? Miasto jako wspólnota komunikacyjna: próba wyboru i oceny podstaw teoretycznych [Thorn im 19. Jahrhundert als Sprach-/Sprachengemeinschaft? Die Stadt als Kommunikationsgemeinschaft: Versuch einer Auswahl und Beurteilung von theoretischen Grundlagen]. In: Dialektologie a geolingvistika v současne středni Evropě, Bd. 2. Opava: Slezská Univerzita, 48-60.

Grotek, Edyta (2018): Miejsca pamięci a toruńska tożsamość zbiorowa w XIX wieku. Przypadek Kopernika [Erinnerungsorte und Thorner kollektive Identität im 19. Jahrhundert. Der Fall von Kopernikus]. In: Czachur, Waldemar (Hg.): Pamięć w ujęciu lingwistycznym. Zagadnienia teoretyczne i metodyczne. Warszawa: Wyd. UW, 159-187.

Halbwachs, Maurice (1985): Das kollektive Gedächtnis. Mit einem Geleitwort zur deutschen Ausgabe von Heinz Maus. Frankfurt/M.: Fischer.

Hoffmann-Axt helm, Dieter (1993): Die dritte Stadt. Frankfurt/M.: Suhrkamp. 
LINDNER, Rolf (2011): Offenheit - Vielfalt - Gestalt. Die Stadt als kultureller Raum. In: JAEger, Friedrich / RÜSEN, Jörn (Hg.): Handbuch der Kulturwissenschaften. Bd. 3: Themen und Tendenzen. Stuttgart, Weimar: Metzler, 385-398.

Loew, Peter Oliver (2006): Gdańsk. Między mitami [Danzig. Unter den Mythen]. Olsztyn: Borussia. Nora, Pierre (1998): Zwischen Geschichte und Gedächtnis. Frankfurt/M.: Fischer.

SANDig, Barbara (2000): Text als prototypisches Konzept. Anwendungsbeispiele, Methodenreflexion, Perspektiven. In: Mangasser-Wahl, Martina (Hg.): Prototypentheorie in der Linguistik. Tübingen: Stauffenburg, 93-112.

Schwarz-Friesel, Monika / Consten, Manfred (2014): Einführung in die Textlinguistik. Darmstadt: WBG.

STIEBNER, Erhardt D. / LeOnhard, Walter (1977): Bruckmann's Handbuch der Schrift. München: Bruckmann.

SzAlEwska, Katarzyna (2017): Urbanalia: miasto i jego teksty [Urbanalia: die Stadt und ihre Texte]. Gdańsk: Słowo/Obraz Terytoria.

WALlIS, Aleksander (1990): Socjologia przestrzeni [Raumsoziologie]. Warszawa: Niezależna Oficyna Wydawnicza. 